TRANSACTIONS OF THE

AMERICAN MATHEMATICAL SOCIETY

Volume 361, Number 1, January 2009, Pages 525-546

S 0002-9947(08)04616-3

Article electronically published on August 18, 2008

\title{
COMPLETE SEGAL SPACES ARISING FROM SIMPLICIAL CATEGORIES
}

\author{
JULIA E. BERGNER
}

\begin{abstract}
In this paper, we compare several functors which take simplicial categories or model categories to complete Segal spaces, which are particularly nice simplicial spaces which, like simplicial categories, can be considered to be models for homotopy theories. We then give a characterization, up to weak equivalence, of complete Segal spaces arising from these functors.
\end{abstract}

\section{INTRODUCTION AND OVERVIEW}

The idea that simplicial categories, or categories enriched over simplicial sets, model homotopy theories goes back to a series of several papers by Dwyer and Kan [8, 10, 11], 12. Taking the viewpoint that a model category, or more generally a category with weak equivalences, can be considered to be a model for a homotopy theory, they develop two methods to obtain from a model category a simplicial category. This "simplicial localization" encodes higher-order structure which is lost when we pass to the homotopy category associated to the model category. Furthermore, they prove that, up to a natural notion of weak equivalence of simplicial categories (see Definition 3.1), every simplicial category arises as the simplicial localization of some category with weak equivalences [10, 2.1]. Thus, the category of all (small) simplicial categories with these weak equivalences can be regarded as the "homotopy theory of homotopy theories".

This notion was mentioned briefly at the end of Dwyer and Spalinski's introduction to model categories [13, 11.6], and was made precise by the author in [2, 1.1], in which the category $\mathcal{S C}$ of small simplicial categories with these weak equivalences is shown to have the structure of a model category.

However, this category is not practically useful for many purposes. Simplicial categories are not particularly easy objects to work with, and the weak equivalences, while natural generalizations of equivalences of categories, are difficult to identify. Motivated by this problem, Rezk defines a model structure, which we denote $\mathcal{C S S}$, on the category of simplicial spaces, in which the fibrant-cofibrant objects are called complete Segal spaces (see Definition 4.3). This model structure is especially nice, in that the objects are just diagrams of simplicial sets and the weak equivalences,

Received by the editors April 23, 2007.

2000 Mathematics Subject Classification. Primary 55U40; Secondary 55U35, 18G55, 18G30, $18 \mathrm{D} 20$

Key words and phrases. Simplicial categories, model categories, complete Segal spaces, homotopy theories.

(C)2008 American Mathematical Society Reverts to public domain 28 years from publication 
at least between complete Segal spaces, are just levelwise weak equivalences of simplicial sets. Furthermore, this model structure has the additional structures of a simplicial model category and a monoidal model category. Because it is given by a localization of a model structure on the category of simplicial spaces with levelwise weak equivalences, it is an example of a presentation for a homotopy theory as described by Dugger [7].

In his paper, Rezk defines two different functors, one from the category of simplicial categories, and one from the category of model categories, to the category of complete Segal spaces. Thus, he describes a relationship between simplicial categories and complete Segal spaces, but he does not give an inverse construction. However, the author was able to show in [5] that the model categories $\mathcal{S C}$ and $\mathcal{C S S}$ are Quillen equivalent to one another, and therefore are models for the same homotopy theory. Thus, the hope is that we can answer questions about simplicial categories by working with complete Segal spaces. This paper is the beginning of that project.

However, the functor that we use to show that the two model categories are Quillen equivalent is not the same as Rezk's functor. Furthermore, the question arises whether Rezk's functor on model categories agrees with the composite of the simplicial localization functor with his functor on simplicial categories. Rezk gives the beginning of a proof that the two functors agree when the model category in question has the additional structure of a simplicial model category. Our goal in this paper is to prove that, up to weak equivalence in $\mathcal{C S S}$, the two functors from $\mathcal{S C}$ to $\mathcal{C S S}$ are the same, that Rezk's result holds for model categories which are not necessarily simplicial, and that this result does imply that the functor on model categories agrees with the one on their corresponding simplicial categories up to weak equivalence.

We then go on to characterize, up to weak equivalence, the complete Segal space arising from any simplicial category. It can be described at each level as the nerve of the monoid of self weak equivalences of representing objects of the category.

We should mention that the model categories $\mathcal{S C}$ and $\mathcal{C S S}$ are only two of several known models for the homotopy theory of homotopy theories. Our proof that the two are Quillen equivalent actually uses two intermediate model structures, $\mathcal{S} e \mathcal{C} a t_{c}$ and $\mathcal{S e C a t}$, on the category of Segal precategories, or simplicial spaces with a discrete space at level zero 5. The fibrant-cofibrant objects in these structures are known as Segal categories. Furthermore, Joyal and Tierney have shown that there is a Quillen equivalence between each of these model structures and a model structure $\mathcal{Q C}$ at on the category of simplicial sets [17, [19]. The fibrant-cofibrant objects of this model structure are known as quasi-categories and are generalizations of Kan complexes [18. One can compare our composite functor $\mathcal{S C} \rightarrow \mathcal{C S S}$ to one they define which factors through $\mathcal{Q C}$ at rather than through $\mathcal{S e C} a t_{c}$ and $\mathcal{S} \mathcal{C} a t_{f}$. It is a consequence of Joyal and Tierney's work that these two different functors give rise to weakly equivalent complete Segal spaces, as we will describe further in the section on complete Segal spaces. An introduction to each of these model structures and the Quillen equivalences can be found in the survey paper [4.

In [1, we use the results of this paper to consider the complete Segal spaces arising from the homotopy fiber product construction for model categories, as described by Toën in his work on derived Hall algebras [24]. It seems that results 
relating diagrams of model categories to diagrams of complete Segal spaces will prove to be useful.

\section{BACKGROUND ON MODEL CATEGORIES AND SIMPLICIAL OBJECTS}

In this section, we summarize some facts about model categories, simplicial sets, and other simplicial objects that we need in the course of this paper.

A model category $\mathcal{M}$ is a category with three distinguished classes of morphisms, fibrations, cofibrations, and weak equivalences. A morphism which is both a (co)fibration and a weak equivalence is called an acyclic (co)fibration. The category $\mathcal{M}$ with these choices of classes is required to satisfy five axioms [13, 3.3].

Axiom MC1 guarantees that $\mathcal{M}$ has small limits and colimits, so in particular $\mathcal{M}$ has an initial object and a terminal object. An object $X$ in a model category $\mathcal{M}$ is fibrant if the unique map $X \rightarrow *$ to the terminal object is a fibration. Dually, $X$ is cofibrant if the unique map from the initial object $\phi \rightarrow X$ is a cofibration.

The factorization axiom (MC5) can be applied in such a way that, given any object $X$ of $\mathcal{M}$, we can factor the map $X \rightarrow *$ as a composite

$$
X \stackrel{\sim}{\longrightarrow} X^{\prime} \longrightarrow *
$$

of an acyclic cofibration followed by a fibration. In this case, $X^{\prime}$ is called a fibrant replacement of $X$, since it is weakly equivalent to $X$ and fibrant. This replacement is not necessarily unique, but in all the model categories we consider here it can be assumed to be functorial [16, 1.1.1]. Cofibrant replacements can be defined dually.

The structure of a model category enables us to invert the weak equivalences formally in such a way that we still have a set, rather than a proper class, of morphisms between any two objects. If we were merely to take the localization $\mathcal{W}^{-1} \mathcal{M}$, there would be no guarantee that we would not have a proper class. However, with the structure of a model category, we can define the homotopy category $\operatorname{Ho}(\mathcal{M})$ to have the same objects as $\mathcal{M}$, and as morphisms

$$
\operatorname{Hom}_{\operatorname{Ho}(\mathcal{M})}(X, Y)=\left[X^{c f}, Y^{c f}\right]_{\mathcal{M}},
$$

where the right-hand side denotes homotopy classes of maps between fibrant-cofibrant replacements of $X$ and $Y$, respectively.

The standard notion of equivalence of model categories is given by the following definitions. First, recall that an adjoint pair of functors $F: \mathcal{C} \leftrightarrows \mathcal{D}: G$ satisfies the property that, for any objects $X$ of $\mathcal{C}$ and $Y$ of $\mathcal{D}$, there is a natural isomorphism

$$
\varphi: \operatorname{Hom}_{\mathcal{D}}(F X, Y) \rightarrow \operatorname{Hom}_{\mathcal{C}}(X, G Y) .
$$

The functor $F$ is called the left adjoint and $G$ the right adjoint [20, IV.1].

Definition 2.1 ([16, 1.3.1]). An adjoint pair of functors $F: \mathcal{M} \leftrightarrows \mathcal{N}: G$ between model categories is a Quillen pair if $F$ preserves cofibrations and $G$ preserves fibrations.

Definition 2.2 ([16, 1.3.12]). A Quillen pair of model categories is a Quillen equivalence if for all cofibrant $X$ in $\mathcal{M}$ and fibrant $Y$ in $\mathcal{N}$, a map $f: F X \rightarrow Y$ is a weak equivalence in $\mathcal{D}$ if and only if the map $\varphi f: X \rightarrow G Y$ is a weak equivalence in $\mathcal{M}$. 
An important example of a model category is that of the standard model structure on the category of simplicial sets $\mathcal{S} \mathcal{S}$ ets. Recall that a simplicial set is a functor $X: \boldsymbol{\Delta}^{o p} \rightarrow \mathcal{S}$ ets, where $\boldsymbol{\Delta}^{o p}$ is the opposite of the category $\boldsymbol{\Delta}$ of finite ordered sets $[n]=\{0 \rightarrow 1 \rightarrow \cdots \rightarrow n\}$ and order-preserving maps between them. We denote the set $X([n])$ by $X_{n}$. In particular in $X$ we have face maps $d_{i}: X_{n} \rightarrow X_{n-1}$ and degeneracy maps $s_{i}: X_{n} \rightarrow X_{n+1}$ for each $0 \leq i \leq n$, satisfying several compatibility conditions. Three particularly useful examples of simplicial sets are the $n$-simplex $\Delta[n]$ and its boundary $\dot{\Delta}[n]$ for each $n \geq 0$, and the boundary with the $k$ th face removed, $V[n, k]$, for each $n \geq 1$ and $0 \leq k \leq n$. Given a simplicial set $X$, we can take its geometric realization $|X|$, which is a topological space [14, I.1].

In the standard model category structure on $\mathcal{S} \mathcal{S}$ ets, the weak equivalences are the maps $f: X \rightarrow Y$ for which the geometric realization $|f|:|X| \rightarrow|Y|$ is a weak homotopy equivalence of topological spaces [14, I.11.3]. In fact, this model structure on simplicial sets is Quillen equivalent to the standard model structure on the category of topological spaces [16, 3.6.7].

More generally, a simplicial object in a category $\mathcal{C}$ is a functor $\boldsymbol{\Delta}^{o p} \rightarrow \mathcal{C}$. The two main examples which we consider in this paper are those of simplicial spaces (also called bisimplicial sets), or functors $\boldsymbol{\Delta}^{o p} \rightarrow \mathcal{S}$ Sets, and simplicial groups. We denote the category of simplicial spaces by $\mathcal{S} \mathcal{S e t s}^{{ }^{o p}}{ }^{o p}$.

A simplicial set $X$ can be regarded as a simplicial space in two ways. It can be considered a constant simplicial space with the simplicial set $X$ at each level, and in this case we will denote the constant simplicial set by $c X$ or just $X$ if no confusion will arise. Alternatively, we can take the simplicial space, which we denote $X^{t}$, for which $\left(X^{t}\right)_{n}$ is the discrete simplicial set $X_{n}$. The superscript $t$ is meant to suggest that this simplicial space is the "transpose" of the constant simplicial space.

A natural choice for the weak equivalences in the category $\mathcal{S} \mathcal{S e t s}^{\boldsymbol{\Delta}^{o p}}$ is the class of levelwise weak equivalences of simplicial sets. If we define the cofibrations to be levelwise also, we obtain a model structure, which is usually referred to as the Reedy model structure on $\mathcal{S S e t s}^{\boldsymbol{\Delta}^{o p}}$ [22].

The Reedy model structure has the additional structure of a simplicial model category. In particular, given any two objects $X$ and $Y$ of $\mathcal{S S e t}^{{ }^{\boldsymbol{o}}{ }^{o p}}$, there is a mapping space, or simplicial set $\operatorname{Map}(X, Y)$ satisfying compatibility conditions 15 , 9.1.6]. In the case where $X$ is cofibrant (as is true of all objects in the Reedy model structure) and $Y$ is fibrant, this choice of mapping space is homotopy invariant.

One way to obtain other model structures on the category of simplicial spaces is to localize the Reedy structure with respect to a set of maps. While this process works for much more general model categories [15, 3.3.1], we will focus here on this particular case. Let $S=\{f: A \rightarrow B\}$ be a set of maps of simplicial spaces. A Reedy fibrant simplicial space $W$ is $S$-local if for each map $f \in S$, the induced map

$$
\operatorname{Map}(f, W): \operatorname{Map}(B, W) \rightarrow \operatorname{Map}(A, W)
$$

is a weak equivalence of simplicial sets. A map $g: X \rightarrow Y$ is then an $S$-local equivalence if for any $S$-local object $W$, the induced map

$$
\operatorname{Map}(g, W): \operatorname{Map}(Y, W) \rightarrow \operatorname{Map}(X, W)
$$

is a weak equivalence of simplicial sets. 
Theorem 2.3 ([15, 4.1.1]). There is a model structure $L_{S} \mathcal{S} \mathcal{S}$ ets ${ }^{{ }^{o p}}$ on the category of simplicial spaces in which

- the weak equivalences are the S-local equivalences,

- the cofibrations are levelwise cofibrations of simplicial sets, and

- the fibrant objects are the $S$-local objects.

Furthermore, this model category has the additional structure of a simplicial model category.

We now turn to a few facts about simplicial groups, or functors from $\boldsymbol{\Delta}^{o p}$ to the category of groups. Given a simplicial group $G$, we can take its nerve, a simplicial space with

$$
\operatorname{nerve}(G)_{n, m}=\operatorname{Hom}\left([m], G_{n}\right) \text {. }
$$

Taking the diagonal of this simplicial space, we obtain a simplicial set, also often called the nerve of $G$.

From another perspective, for $G$ a simplicial group (or, more generally, a simplicial monoid), we can find a classifying complex of $G$, a simplicial set whose geometric realization is the classifying space $B G$. A precise construction can be made for this classifying space by the $\bar{W} G$ construction [14, V.4.4], 21. However, we are not so concerned here with the precise construction as with the fact that such a classifying space exists, so for simplicity we will simply write $B G$ for the classifying complex of $G$.

\section{Simplicial CATEgORIES AND SIMPlicial LOCALIZATIONS}

In this section, we consider simplicial categories and show how they arise from Dwyer and Kan's simplicial localization techniques. We then discuss model category structures, first on the category of simplicial categories with a fixed object set, and then on the category of all small simplicial categories.

First of all, we clarify some terminology. In this paper, by (small) "simplicial category" we will mean a category with a set of objects and a simplicial set of morphisms $\operatorname{Map}(x, y)$ between any two objects $x$ and $y$, also known as a category enriched over simplicial sets. This notion does not coincide with the more general one of a simplicial object in the category of small categories, in which we would also have a simplicial set of objects. Using this more general definition, if we impose the additional condition that all face and degeneracy maps are the identity on the objects, then we get our more restricted notion.

A simplicial category can be seen as a generalization of a category, since any ordinary category can be regarded as a simplicial category with a discrete mapping space. Given any simplicial category $\mathcal{C}$, we can consider its category of components $\pi_{0} \mathcal{C}$, which has the same objects as $\mathcal{C}$ and whose morphisms are given by

$$
\operatorname{Hom}_{\pi_{0} \mathcal{C}}(x, y)=\pi_{0} \operatorname{Map}_{\mathcal{C}}(x, y) \text {. }
$$

The following definition of weak equivalence of simplicial categories is a natural generalization of the notion of equivalence of categories.

Definition 3.1. A simplicial functor $f: \mathcal{C} \rightarrow \mathcal{D}$ is a Dwyer-Kan equivalence or $D K$-equivalence if the following two conditions hold:

(1) For any objects $x$ and $y$ of $\mathcal{C}$, the induced map $\operatorname{Map}(x, y) \rightarrow \operatorname{Map}(f x, f y)$ is a weak equivalence of simplicial sets. 
(2) The induced map on the categories of components $\pi_{0} f: \pi_{0} \mathcal{C} \rightarrow \pi_{0} \mathcal{D}$ is an equivalence of categories.

The idea of obtaining a simplicial category from a model category $\mathcal{M}$ goes back to several papers of Dwyer and Kan [8, [11, 12. In fact, they define two different methods of doing so, the simplicial localization $L \mathcal{M}[12$ and the hammock localization $L^{H} \mathcal{M}$ 11. The first has the advantage of being easier to describe, while the second is more convenient for making calculations.

It should be noted that these constructions can be made for more general categories with weak equivalences and do not depend on the model structure if we are willing to ignore the potential set-theoretic difficulties. However, as with the homotopy category construction, the hammock localization in particular can be defined much more nicely when we have the additional structure of a model category.

We begin with the construction of the simplicial localization $L \mathcal{M}$. Recall that, given a category $\mathcal{M}$ with some choice of weak equivalences $\mathcal{W}$, we denote the localization of $\mathcal{M}$ with respect to $\mathcal{W}$ by $\mathcal{W}^{-1} \mathcal{M}$. This localization is obtained from $\mathcal{M}$ by formally inverting the maps of $\mathcal{W}$. Further, recall that, given a category $\mathcal{M}$, we denote by $F \mathcal{M}$ the free category on $\mathcal{M}$, or category with the same objects as $\mathcal{M}$ and morphisms freely generated by the nonidentity morphisms of $\mathcal{M}$. Note in particular that there are natural functors $F \mathcal{M} \rightarrow \mathcal{M}$ and $F \mathcal{M} \rightarrow F^{2} \mathcal{M}$ [12, 2.4]. These functors can be used to define a simplicial resolution $F_{*} \mathcal{M}$, which is a simplicial category with the category $F^{k+1} \mathcal{M}$ at level $k$ [12, 2.5].

We can apply this same construction to the subcategory $\mathcal{W}$ to obtain a simplicial resolution $F_{*} \mathcal{W}$. Using these two resolutions, we have the following definition.

Definition $3.2([12,4.1])$. The simplicial localization of $\mathcal{M}$ with respect to $\mathcal{W}$ is the localization $\left(F_{*} \mathcal{W}\right)^{-1}\left(F_{*} \mathcal{M}\right)$. This simplicial localization is denoted $L(\mathcal{M}, \mathcal{W})$ or simply $L \mathcal{M}$.

The following result gives interesting information about the mapping spaces in $L \mathcal{M}$ in the case where $\mathcal{W}$ is all of $\mathcal{M}$.

Proposition $3.3([12,5.5])$. Suppose that $\mathcal{W}=\mathcal{M}$ and nerve $(\mathcal{M})$ is connected.

(i) The simplicial localization $L \mathcal{M}$ is a simplicial groupoid, so for all objects $x$ and $y$, the simplicial sets $\operatorname{Map}_{L \mathcal{M}}(x, y)$ are all isomorphic. In particular, the simplicial sets $\operatorname{Map}_{L \mathcal{M}}(x, x)$ are all isomorphic simplicial groups.

(ii) The classifying complex $B \operatorname{Map}_{L \mathcal{M}}(x, x)$ has the homotopy type of nerve $(\mathcal{M})$, and thus each simplicial set $\operatorname{Map}_{L \mathcal{M}}(x, y)$ has the homotopy type of the loop space $\Omega(\operatorname{nerve}(\mathcal{M}))$.

We now turn to the other construction, that of the hammock localization. Again, let $\mathcal{M}$ be a category with a specified subcategory $\mathcal{W}$ of weak equivalences.

Definition 3.4 ([11, 3.1]). The hammock localization of $\mathcal{M}$ with respect to $\mathcal{W}$, denoted $L^{H}(\mathcal{M}, \mathcal{W})$, or simply $L^{H} \mathcal{M}$, is the simplicial category defined as follows:

(1) The simplicial category $L^{H} \mathcal{M}$ has the same objects as $\mathcal{M}$.

(2) Given objects $X$ and $Y$ of $\mathcal{M}$, the simplicial set $\operatorname{Map}_{L^{H} \mathcal{M}}(X, Y)$ has as $k$-simplices the reduced hammocks of width $k$ and any length between $X$ 
and $Y$, or commutative diagrams of the form

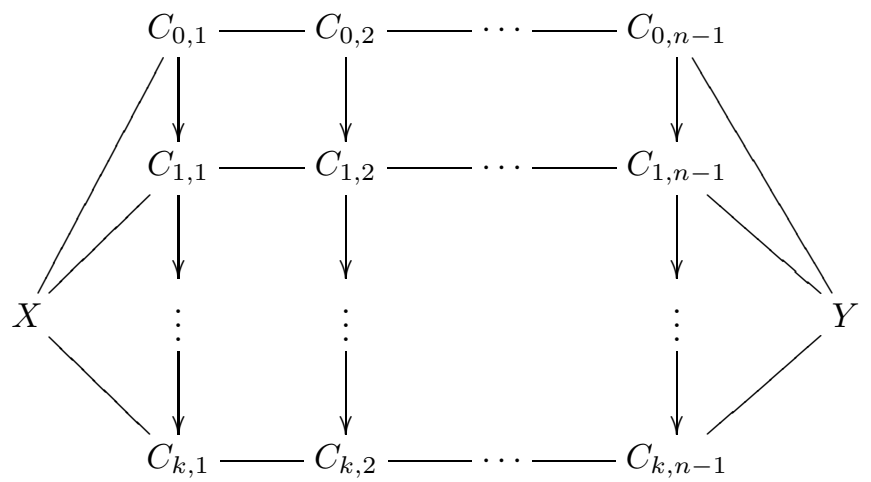

in which

(i) the length of the hammock is any integer $n \geq 0$,

(ii) the vertical maps are all in $\mathcal{W}$,

(iii) in each column all the horizontal maps go in the same direction, and if they go to the left, then they are in $\mathcal{W}$,

(iv) the maps in adjacent columns go in opposite directions, and

(v) no column contains only identity maps.

Proposition 3.5 ([8, 2.2]). For a given model category $\mathcal{M}$, the simplicial categories $L \mathcal{M}$ and $L^{H} \mathcal{M}$ are DK-equivalent.

We should add that the description of the hammock localization can be greatly simplified if we make use of the model category structure on $\mathcal{M}$. In this case, Dwyer and Kan prove that it suffices to consider hammocks of length 3 such as the following [8, $\S 8]$ :

$$
X \stackrel{\simeq}{\longleftarrow} C_{0,1} \longrightarrow C_{0,2} \stackrel{\simeq}{\longleftarrow} Y .
$$

Restricting to the category of simplicial categories with a fixed set $\mathcal{O}$ of objects, Dwyer and Kan prove the existence of a model structure on this category, which we denote $\mathcal{S C}_{\mathcal{O}}[12,7.2]$. In this situation, the weak equivalences are the DK-equivalences, but with the objects fixed the second condition follows immediately from the first. The fibrations in this model structure are given by the functors $f: \mathcal{C} \rightarrow \mathcal{D}$ inducing, for any objects $x$ and $y$, fibrations of simplicial sets $\operatorname{Map}_{\mathcal{C}}(x, y) \rightarrow \operatorname{Map}_{\mathcal{D}}(x, y)$.

The cofibrations are then defined to be the maps with the left lifting property with respect to the acyclic fibrations. However, they can be more precisely characterized. To do so, we recall the definition of a free map of simplicial categories.

Definition 3.6 $([12,7.4])$. A map $f: \mathcal{C} \rightarrow \mathcal{D}$ in $\mathcal{S C}_{\mathcal{O}}$ is free if

(1) $f$ is a monomorphism,

(2) if $*$ denotes the free product, then in each simplicial dimension $k$, the category $\mathcal{D}_{k}$ admits a unique free factorization $\mathcal{D}_{k}=f\left(\mathcal{C}_{k}\right) * \mathcal{F}_{k}$, where $\mathcal{F}_{k}$ is a free category, and

(3) for each $k \geq 0$, all degeneracies of generators of $\mathcal{F}_{k}$ are generators of $\mathcal{F}_{k+1}$. 
Definition $3.7([12,7.5])$. A map $f: \mathcal{C} \rightarrow \mathcal{D}$ of simplicial categories is a strong retract of a map $f^{\prime}: \mathcal{C} \rightarrow \mathcal{D}^{\prime}$ if there exists a commutative diagram

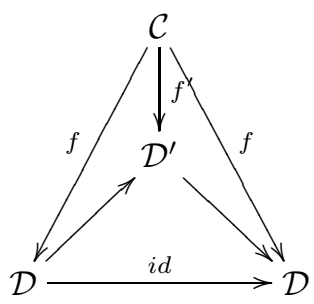

Using these definitions, Dwyer and Kan prove the following result.

Proposition $3.8([12,7.6])$. The cofibrations of $\mathcal{S C}_{\mathcal{O}}$ are precisely the strong retracts of free maps. In particular, a cofibrant simplicial category is a retract of a free category.

This result can then be generalized to the category of all simplicial categories, in which the DK-equivalences are the weak equivalences. If $\mathcal{C}$ is a simplicial category, a morphism $e \in \operatorname{Hom}_{\mathcal{C}}(a, b)_{0}$ is a homotopy equivalence if it becomes an isomorphism in $\pi_{0} \mathcal{C}$.

Theorem 3.9 ([2, 1.1]). There is a model category structure $\mathcal{S C}$ on the category of small simplicial categories in which

- the weak equivalences are the Dwyer-Kan equivalences, and

- the fibrations are the maps $f: \mathcal{C} \rightarrow \mathcal{D}$ satisfying the following two conditions:

(i) For any objects $a_{1}$ and $a_{2}$ in $\mathcal{C}$, the map

$$
\operatorname{Hom}_{\mathcal{C}}\left(a_{1}, a_{2}\right) \rightarrow \operatorname{Hom}_{\mathcal{D}}\left(f a_{1}, f a_{2}\right)
$$

is a fibration of simplicial sets.

(ii) For any object $a_{1}$ in $\mathcal{C}, b$ in $\mathcal{D}$, and homotopy equivalence $e: f a_{1} \rightarrow b$ in $\mathcal{D}$, there is an object $a_{2}$ in $\mathcal{C}$ and homotopy equivalence $d: a_{1} \rightarrow a_{2}$ in $\mathcal{C}$ such that $f d=e$.

\section{Complete Segal SPaces}

Here we define complete Segal spaces and describe Rezk's model structure on the category of simplicial spaces, in which the complete Segal spaces are the fibrantcofibrant objects.

Recall that by a simplicial space we mean a simplicial object in the category of simplicial sets, or a functor $\boldsymbol{\Delta}^{o p} \rightarrow \mathcal{S} \mathcal{S}$ ets. In section 2 , we described the Reedy model category structure on this category, in which both the weak equivalences and cofibrations are defined levelwise. The model structure $\mathcal{C S S}$ is given by a localization of this structure with respect to a set of maps.

We begin with the definition of a Segal space. In [23, 4.1], Rezk defines for each $0 \leq i \leq n-1$ a map $\alpha^{i}:[1] \rightarrow[n]$ in $\boldsymbol{\Delta}$ such that $0 \mapsto i$ and $1 \mapsto i+1$. There is a corresponding map $\alpha_{i}: \Delta[1] \rightarrow \Delta[n]$. Then for each $n$ he defines the simplicial space

$$
G(n)^{t}=\bigcup_{i=0}^{n-1} \alpha_{i} \Delta[1]^{t} \subset \Delta[n]^{t} .
$$


Let $X$ be a Reedy fibrant simplicial space. There is a weak equivalence of simplicial sets

$$
\operatorname{Map}_{\mathcal{S S e t}_{c}^{\Delta_{c}^{o p}}}\left(G(n)^{t}, X\right) \rightarrow \underbrace{X_{1} \times_{X_{0}} \cdots \times_{X_{0}} X_{1}}_{n},
$$

where the right-hand side is the limit of the diagram

$$
X_{1} \stackrel{d_{0}}{\longrightarrow} X_{0} \stackrel{d_{1}}{\longleftarrow} X_{1} \stackrel{d_{0}}{\longrightarrow} \cdots \stackrel{d_{0}}{\longrightarrow} X_{0} \stackrel{d_{1}}{\longleftarrow} X_{1}
$$

with $n$ copies of $X_{1}$.

Now, given any $n$, define the map $\varphi^{n}: G(n)^{t} \rightarrow \Delta[n]^{t}$ to be the inclusion map. Then for any Reedy fibrant simplicial space $W$ there is a map

$$
\varphi_{n}=\operatorname{Map}_{\mathcal{S S e t s} s_{c}^{\Delta o p}}\left(\varphi^{n}, W\right): \operatorname{Map}_{\mathcal{S S e t s _ { c }} \Delta_{c}^{o p}}\left(\Delta[n]^{t}, W\right) \rightarrow \operatorname{Map}_{\mathcal{S} \operatorname{Sets}_{c}^{\Delta o p}}\left(G(n)^{t}, W\right) .
$$

More simply written, this map is

$$
\varphi_{n}: W_{n} \rightarrow \underbrace{W_{1} \times_{W_{0}} \cdots \times_{W_{0}} W_{1}}_{n}
$$

and is often called a Segal map. The Segal map is actually defined for any simplicial space $W$, but here we assume Reedy fibrancy so that the mapping spaces involved are homotopy invariant.

Definition $4.1(23,4.1])$. A Reedy fibrant simplicial space $W$ is a Segal space if for each $n \geq 2$ the Segal map

$$
\varphi_{n}: W_{n} \rightarrow W_{1} \times_{W_{0}} \cdots \times_{W_{0}} W_{1}
$$

is a weak equivalence of simplicial sets.

In fact, there is a model category structure $\mathcal{S} S \mathcal{S} p$ on the category of simplicial spaces in which the fibrant objects are precisely the Segal spaces [23, 7.1]. This model structure is obtained from the Reedy structure via localization.

The idea is that in a Segal space there is a notion of "composition", at least up to homotopy. In fact, given a Segal space, we can sensibly use many categorical notions. We summarize some of these ideas here; a detailed description is given by Rezk 23. The objects of a Segal space $W$ are given by the set $W_{0,0}$. Given the map

$$
\left(d_{1}, d_{0}\right): W_{1} \rightarrow W_{0} \times W_{0},
$$

the mapping space $\operatorname{map}_{W}(x, y)$ is given by the fiber of this map over $(x, y)$. (The fact that $W$ is Reedy fibrant guarantees that this mapping space is homotopy invariant.) Two maps $f, g \in \operatorname{map}_{W}(x, y)_{0}$ are homotopic if they lie in the same component of the simplicial set $\operatorname{map}_{W}(x, y)$. Thus, we define the space of homotopy equivalences $W_{\text {hoequiv }} \subseteq W_{1}$ to consist of all the components containing homotopy equivalences.

Given any $\left(x_{0}, \ldots, x_{n}\right) \in W_{0,0}^{n+1}$, let $\operatorname{map}_{W}\left(x_{0}, \ldots, x_{n}\right)$ denote the fiber of the map

$$
\left(\alpha_{0}, \ldots, \alpha_{n}\right): W_{n} \rightarrow W_{0}^{n+1}
$$

over $\left(x_{0}, \ldots, x_{n}\right)$. Consider the commutative diagram

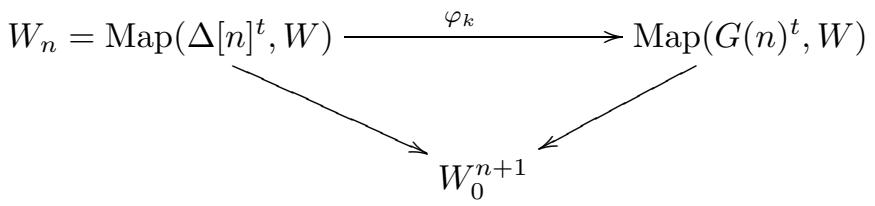


and notice that, since $W$ is a Segal space, the horizontal arrow is a weak equivalence and a fibration. In particular, this map induces an acyclic fibration on the fibers of the two vertical arrows,

$$
\operatorname{map}_{W}\left(x_{0}, \ldots, x_{n}\right) \rightarrow \operatorname{map}_{W}\left(x_{n-1}, x_{n}\right) \times \cdots \times \operatorname{map}_{W}\left(x_{0}, x_{1}\right) .
$$

Given $f \in \operatorname{map}(x, y)_{0}$ and $g \in \operatorname{map}(y, z)_{0}$, their composite is a lift of $(g, f) \in$ $\operatorname{map}(y, z) \times \operatorname{map}(x, y)$ along $\varphi_{2}$ to some $k \in \operatorname{map}(x, y, z)_{0}$. The result of this composition is defined to be $d_{1}(k) \in \operatorname{map}(x, z)_{0}$. It can be shown that any two results are homotopic, so we can use $g \circ f$ unambiguously.

Then, the homotopy category of $W$, denoted $\operatorname{Ho}(W)$, has as objects the elements of the set $W_{0,0}$, and

$$
\operatorname{Hom}_{H o(W)}(x, y)=\pi_{0} \operatorname{map}_{W}(x, y) .
$$

A homotopy equivalence in $W$ is a 0 -simplex of $W_{1}$ whose image in $\operatorname{Ho}(W)$ is an isomorphism.

Definition 4.2. A map $f: W \rightarrow Z$ of Segal spaces is a Dwyer-Kan equivalence if

(1) for any objects $x$ and $y$ of $W$, the induced $\operatorname{map}_{\operatorname{map}_{W}}(x, y) \rightarrow \operatorname{map}_{Z}(f x, f y)$ is a weak equivalence of simplicial sets, and

(2) the induced map $\operatorname{Ho}(W) \rightarrow \operatorname{Ho}(Z)$ is an equivalence of categories.

Notice that the definition of these maps bears a striking resemblance to that of the Dwyer-Kan equivalences between simplicial categories, hence the use of the same name.

For a Segal space $W$, note that the degeneracy map $s_{0}: W_{0} \rightarrow W_{1}$ factors through the space of homotopy equivalences $W_{\text {hoequiv }}$, since the image of $s_{0}$ consists of "identity maps". Given this fact, we are now able to give a definition of a complete Segal space.

Definition $4.3([23, \S 6])$. A Segal space $W$ is a complete Segal space if the map $W_{0} \rightarrow W_{\text {hoequiv }}$ given above is a weak equivalence of simplicial sets.

The idea behind this notion is that, although $W_{0}$ is not required to be discrete, as the objects are for a simplicial category, it is not heuristically too different from a simplicial space with discrete 0-space. (This viewpoint is further confirmed by the comparison of complete Segal spaces with Segal categories, which are essentially the analogues of Segal spaces with discrete 0-space [5, 6.3].)

Now, we give a description of the model structure $\mathcal{C S S}$. We do not give all the details here, such as a description of an arbitrary weak equivalence, but refer the interested reader to Rezk's paper [23, §7].

Theorem $4.4([23,7.2])$. There is a model structure $\mathcal{C S S}$ on the category of simplicial spaces, obtained as a localization of the Reedy model structure, such that

(1) the fibrant objects are precisely the complete Segal spaces,

(2) the cofibrations are the monomorphisms; in particular, every object is cofibrant,

(3) the weak equivalences between Segal spaces are Dwyer-Kan equivalences, and

(4) the weak equivalences between complete Segal spaces are levelwise weak equivalences of simplicial sets. 
Furthermore, $\mathcal{C S S}$ has the additional structure of a simplicial model category and is cartesian closed.

The fact that $\mathcal{C S S}$ is cartesian closed allows us to consider, for any complete Segal space $W$ and simplicial space $X$, the complete Segal space $W^{X}$. In particular, using the simplicial structure, the simplicial set at level $n$ is given by

$$
\left(W^{X}\right)_{n}=\operatorname{Map}\left(X \times \Delta[n]^{t}, W\right) .
$$

If $W$ is a (not necessarily complete) Segal space, then $W^{X}$ is again a Segal space; in other words, the model category $\mathcal{S} e \mathcal{S} p$ is also cartesian closed.

We denote the functorial fibrant replacement functor in $\mathcal{C S S}$ by $L_{\mathcal{C S S}}$. Thus, given any simplicial space $X$, there is a weakly equivalent complete Segal space $L_{\mathcal{C S S}} X$.

This model structure is connected to the model structure $\mathcal{S C}$ by a chain of Quillen equivalences as follows. Each of these model categories is Quillen equivalent to a model structure $\mathcal{S} e \mathcal{C} a t_{f}$ on the category of Segal precategories. A Segal precategory is a simplicial space $X$ with $X_{0}$ a discrete space. A Segal category is then a Segal precategory with the Segal maps weak equivalences. In the model structure $\mathcal{S e C} a t_{f}$, the fibrant objects are Segal categories, and so it is considered a Segal category model structure on the category of Segal precategories. We have the following chain of Quillen equivalences, with the left adjoint functors topmost:

$$
\mathcal{S C} \leftrightarrows \mathcal{S e C} a t_{f} \rightleftarrows \mathcal{C S S}
$$

The right adjoint $\mathcal{S C} \rightarrow \mathcal{S e C} a t_{f}$ is given by the nerve functor, and the left adjoint $\mathcal{S e C a t} \rightarrow \mathcal{C S S}$ is given by the inclusion functor.

There is actually another chain of Quillen equivalences connecting the two model structures; in this case, both $\mathcal{S C}$ and $\mathcal{C S S}$ are Quillen equivalent to Joyal's model structure $\mathcal{Q C}$ at on the category of simplicial sets [18. The fibrant objects in $\mathcal{Q C}$ at are quasi-categories, or simplicial sets $K$ such that a dotted arrow lift exists making the diagram

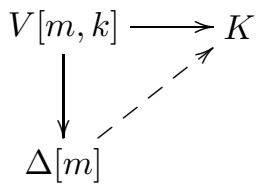

commute for any $0<k<m$. The chain of Quillen equivalences in this case is given by

$$
\mathcal{S C} \rightleftarrows \mathcal{Q C} \text { at } \leftrightarrows \mathcal{C S S}
$$

The right adjoint $\mathcal{S C} \rightarrow \mathcal{Q C}$ at is given by Cordier and Porter's coherent nerve functor [6], [17, 2.10], and the right adjoint $\mathcal{C S S} \rightarrow \mathcal{Q C}$ at is given by sending a simplicial space $W$ to the simplicial set $W_{*, 0}$ [19,4.11]. It is a consequence of work of Joyal [17, §1-2] and of Joyal and Tierney [19, §4-5] that the simplicial space obtained from a simplicial category via these functors is weakly equivalent to the one obtained from the composite functor described in the previous paragraph.

\section{Obtaining COMPlete SEgal SPACES From Simplicial CATEGORIES AND MODEL CATEGORIES}

In this section, we describe several different ways of obtaining a complete Segal space. First, we look at a particularly nice functor which Rezk uses to modify 
the notion of a nerve of a category. Then we look at how this functor can be generalized to one on any simplicial category, and how a similar idea can be used to get a complete Segal space from any model category. We then consider the functors used in the Quillen equivalences connecting $\mathcal{S C}$ and $\mathcal{C S S}$.

Let us begin with Rezk's classifying diagram construction, which associates to any small category $\mathcal{C}$ a complete Segal space $N \mathcal{C}$. First, we denote by nerve $(\mathcal{C})$ the ordinary nerve, which is the simplicial set given by $(\text { nerve }(\mathcal{C}))_{n}=\operatorname{Hom}([n], \mathcal{C})$. Further, we denote by iso $(\mathcal{C})$ the maximal subgroupoid of $\mathcal{C}$, or subcategory of $\mathcal{C}$ with all objects of $\mathcal{C}$ and whose only morphisms are the isomorphisms of $\mathcal{C}$. By $\mathcal{C}^{[n]}$ we denote the category of functors $[n] \rightarrow \mathcal{C}$, or the category whose objects are $n$-chains of composable morphisms in $\mathcal{C}$.

Definition $5.1([23,3.5])$. The classifying diagram $N \mathcal{C}$ is the simplicial space given by $(N \mathcal{C})_{n}=\operatorname{nerve}\left(\operatorname{iso}\left(\mathcal{C}^{[n]}\right)\right)$.

Thus, $(N \mathcal{C})_{0}$ is simply the nerve of iso $(\mathcal{C})$, and $(N \mathcal{C})_{1}$ is the nerve of the maximal subgroupoid of the morphism category of $\mathcal{C}$. In particular, information about invertible morphisms of $\mathcal{C}$ is encoded at level 0 , while information about the other morphisms of $\mathcal{C}$ does not appear until level 1.

Thus, the classifying diagram of a category can be regarded as a more refined version of the nerve, since, unlike the ordinary nerve construction, it enables one to recover information about whether morphisms are invertible or not. This construction is also particularly useful for our purposes due to the following result.

Proposition 5.2 ([23, 6.1]). If $\mathcal{C}$ is a small category, then its classifying diagram $N C$ is a complete Segal space.

However, this construction, as defined above, cannot be used to assign a complete Segal space to any simplicial category, since, beginning with level 1, we would have homotopy invariance problems with a simplicial set of objects in $\mathcal{C}^{[1]}$. Rezk defines an analogous functor, though, from the category of small simplicial categories, which is similar in spirit to the classifying diagram but avoids these difficulties.

Let $I[m]$ denote the category with $m+1$ objects and a single isomorphism between any two objects, and let $E(m)=\operatorname{nerve}(I[m])^{t}$. If $W$ is a Segal space and $X$ is any simplicial space, recall that $W^{X}$ denotes the internal hom object, which is a Segal space. With this notation in place, we can give the definition of Rezk's completion functor. Let $W$ be a Segal space. Then its completion $\widehat{W}$ is defined as a fibrant replacement in $\mathcal{C S S}$ of the simplicial space $\widetilde{W}$ defined by

$$
\widetilde{W}_{n}=\operatorname{diag}\left([m] \mapsto \operatorname{Map}_{S S e t s^{\Delta}} \Delta^{o p}\left(E(m), W^{\Delta[n]^{t}}\right)\right)=\operatorname{diag}\left([m] \mapsto\left(W^{E(m)}\right)_{n}\right) .
$$

From a simplicial category $\mathcal{C}$, then, we can take its nerve to obtain a simplicial space, followed by a fibrant replacement functor in the Segal space model structure, to obtain a Segal space $W$. From $W$ we can then pass to a complete Segal space via this completion functor. We will denote this complete Segal space $L_{C}(W)$, or $L_{C}(\mathcal{C})$, where $W$ comes from a simplicial category as just described.

The first important fact about this completion functor is that the completion map $i_{W}: W \rightarrow \widehat{W}=L_{C}(W)$ is not only a weak equivalence in the model category $\mathcal{C S S}$, but is also a Dwyer-Kan equivalence of Segal spaces [23, §14]. Furthermore, this completion functor restricts nicely to the classifying diagram in the case where $\mathcal{C}$ is a discrete category. 
Proposition $5.3([23,14.2])$. If $\mathcal{C}$ is a discrete category, then $L_{C}(\mathcal{C})$ is isomorphic to $N \mathcal{C}$.

If we begin with a model category $\mathcal{M}$ with a subcategory of weak equivalences $\mathcal{W}$, a functor analogous to the classifying diagram functor can be used to obtain a complete Segal space. In this case, rather than taking the subcategory iso $(\mathcal{M})$ of isomorphisms of $\mathcal{M}$, we take the subcategory of weak equivalences, denoted we $(\mathcal{M})$. Thus, Rezk defines the classification diagram of $(\mathcal{M}, \mathcal{W})$, denoted $N(\mathcal{M}, \mathcal{W})$, by

$$
N(\mathcal{M}, \mathcal{W})_{n}=\operatorname{nerve}\left(\operatorname{we}\left(\mathcal{M}^{[n]}\right)\right) .
$$

Unlike the classifying diagram, the classification diagram of a model category is not necessarily a complete Segal space as stated, but taking a Reedy fibrant replacement of it results in a complete Segal space, as we show in the next section.

Lastly, we have the two functors given by the two different chains of Quillen equivalences between the model categories $\mathcal{S C}$ and $\mathcal{C S S}$. As mentioned in the previous section, these two functors are equivalent. In each case, the resulting simplicial space is not Reedy fibrant in general, and so not a complete Segal space, but applying the fibrant replacement functor $L_{\mathcal{C S S}}$ results in a complete Segal space.

The first of these composite functors, in particular, is simple to describe abstractly, as in the previous section, but it has a disadvantage over Rezk's functor in that it gives very little insight into what the resulting complete Segal space looks like. In the next section, we prove that the two functors from $\mathcal{S C}$ to $\mathcal{C S S}$ result in weakly equivalent complete Segal spaces, and that if we use Rezk's classification diagram construction we get a weakly equivalent complete Segal space to the one we would obtain by taking the simplicial localization followed by his completion functor. We then use Rezk's functor to describe what the complete Segal space corresponding to a simplicial category looks like.

\section{Comparison of FunCtors from $\mathcal{S C}$ to $\mathcal{C S S}$}

Here we prove that each of the functors we have described all give rise to complete Segal spaces weakly equivalent to those given in the previous section. We begin by stating the result that establishes the equivalence between Rezk's completion functor $L_{C}: \mathcal{S C} \rightarrow \mathcal{C S S}$ and the functor arising from the chain of Quillen equivalences factoring through $\mathcal{S} e \mathcal{C} a t_{f}$. Let $L_{\mathcal{C S S}}$ denote the functorial fibrant replacement functor in $\mathcal{C S S}$.

Theorem 6.1. If $\mathcal{C}$ is a simplicial category, then the complete Segal spaces $L_{C}(\mathcal{C})$ and $L_{\mathcal{C S S}}($ nerve $(\mathcal{C}))$ are weakly equivalent in $\mathcal{C S S}$.

Proof. Let $L_{S}$ denote a fibrant replacement functor in the Segal space model structure $\mathcal{S} e \mathcal{S} p$ on the category of simplicial spaces. The fact that the two functors in question result in weakly equivalent complete Segal spaces can be shown by considering the following chain of weak equivalences:

$$
L_{\mathcal{C S S}}(\text { nerve }(\mathcal{C})) \leftarrow \operatorname{nerve}(\mathcal{C}) \rightarrow L_{S} \text { nerve }(\mathcal{C}) \rightarrow L_{C}(\mathcal{C}) .
$$

The map on the left is the localization functor in $\mathcal{C S S}$ and so is a weak equivalence in $\mathcal{C S S}$. The middle map is a weak equivalence in $\mathcal{S} e \mathcal{S} p$ and therefore also a weak equivalence in $\mathcal{C S S}$, since the latter model category is a localization of the former. The map on the right is Rezk's completion, and it is a weak equivalence in $\mathcal{C S S}$, as given in the previous section. Therefore, the objects at the far left and right of this 
zigzag, both of which are complete Segal spaces, are weakly equivalent as objects of $\mathcal{C S S}$.

Now, we would like to compare either of these functors to the classifying diagram construction for a model category $\mathcal{M}$. In other words, we want to show that $N(\mathcal{M}, \mathcal{W})$ is equivalent to $L_{C}\left(L^{H} \mathcal{M}\right)$, where we first take the hammock localization of $\mathcal{M}$ to obtain a simplicial category, and then apply Rezk's functor $L_{C}$.

An initial problem here is that $N(\mathcal{M}, \mathcal{W})$ is not necessarily Reedy fibrant, and so it is not necessarily a complete Segal space. We prove that a Reedy fibrant replacement of it, denoted $N(\mathcal{M}, \mathcal{W})^{f}$, is in fact a complete Segal space in the process of comparing the "mapping spaces" in this Reedy fibrant replacement to the mapping spaces of the hammock localization $L^{H} \mathcal{M}$.

Theorem 6.2. Let $\mathcal{M}$ be a model category, and let $\mathcal{W}$ denote its subcategory of weak equivalences. Then $N(\mathcal{M}, \mathcal{W})^{f}$ is a complete Segal space. Furthermore, for

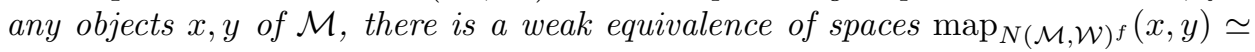
$\operatorname{Map}_{L^{H} \mathcal{M}}(x, y)$, and there is an equivalence of categories $\operatorname{Ho}\left(N(\mathcal{M}, \mathcal{W})^{f}\right) \approx \operatorname{Ho}(\mathcal{M})$.

This result was proved by Rezk in the case where $\mathcal{M}$ is a simplicial model category [23, 8.3], namely, in the case where we do not need to pass to the simplicial localization of $\mathcal{M}$ to consider its function complexes. However, here we prove that, as he conjectured [23, 8.4], the result continues to hold in this more general case.

We prove this theorem very similarly to the way Rezk proves it in the more restricted case, using a proposition of Dwyer and Kan. To begin, we introduce some terminology. Let $\mathcal{M}$ be a model category. A classification complex of $\mathcal{M}$, as defined in [9, 1.2], is the nerve of any subcategory $\mathcal{C}$ of $\mathcal{M}$ such that

(1) every map in $\mathcal{C}$ is a weak equivalence,

(2) if $f: X \rightarrow Y$ in $\mathcal{M}$ is a weak equivalence and either $X$ or $Y$ is in $\mathcal{C}$, then $f$ is in $\mathcal{C}$, and

(3) $\operatorname{nerve}(\mathcal{C})$ is homotopically small; i.e., each homotopy group of $|\operatorname{nerve}(\mathcal{C})|$ is small [11, 2.2].

The special classification complex $\operatorname{sc}(X)$ of an object $X$ in $\mathcal{M}$ is a connected classification complex containing $X$.

Let $\mathcal{M}$ be a model category and $X$ a fibrant-cofibrant object of $\mathcal{M}$. Denote by $\operatorname{Aut}^{h}(X)$ the simplicial monoid of weak equivalences given by $\operatorname{Aut}_{L^{H} \mathcal{M}}^{h}(X)$ in the hammock localization $L^{H} \mathcal{M}$, and by $B \operatorname{Aut}^{h}(X)$ its classifying complex.

The following proposition was proved by Dwyer and Kan in [9, 2.3] in the case that $\mathcal{M}$ is a simplicial model category. However, the proof does not actually require the simplicial structure; in fact, their proof is essentially the one given below, with the extra step showing that the mapping spaces in the hammock localization are equivalent to those given by the simplicial structure of $\mathcal{M}$ [11, 4.8].

Proposition 6.3. Let $X$ be an object of a model category $\mathcal{M}$. The classifying complex BAut ${ }^{h}(X)$ is weakly equivalent to the special classification complex of $X$, $\operatorname{sc}(X)$, and the two can be connected by a finite zigzag of weak equivalences.

Proof. Let $\mathcal{W}$ be the subcategory of weak equivalences of $\mathcal{M}$. Consider the connected component of nerve $(\mathcal{W})$ containing $X$. For the rest of this proof, we assume that $\mathcal{W}$ is such that its nerve is connected. We further assume that nerve $(\mathcal{W})$ is homotopically small, taking an appropriate subcategory, as described in [11, 2.3], if necessary. 
In this case, by Proposition 3.3. the function complexes $\operatorname{Map}_{L \mathcal{W}}(X, X)$ are all isomorphic. Furthermore, by the same result, the classifying complex $B \operatorname{Map}_{L \mathcal{W}}(X, X)$ has the homotopy type of nerve $(\mathcal{W})$. Thus, we can take nerve $(\mathcal{W})$ as $\operatorname{sc}(X)$.

Now, as in the statement of the proposition, we take $\operatorname{Aut}^{h}(X)$ to consist of the components of $\operatorname{Map}_{L^{H} \mathcal{M}}(X, X)$ which are invertible in $\pi_{0} \operatorname{map} L^{H} \mathcal{M}(X, X)$. But, by [11, 4.6(ii)], the map $B \operatorname{Map}_{L^{H} \mathcal{W}}(X, X) \rightarrow B \operatorname{Aut}^{h}(X)$ is a weak equivalence of simplicial sets. Since $L^{H} \mathcal{W}$ can be connected to $L \mathcal{W}$ by a finite string of weak equivalences, it follows that so can $\operatorname{Map}_{L^{H} \mathcal{W}}(X, X)$ and $\operatorname{Map}_{L \mathcal{W}}(X, X)$. Thus, $B \operatorname{Map}_{L \mathcal{W}}(X, X)$ and $B \operatorname{Aut}^{h}(X)$ can also be connected by such a string. It follows that $\operatorname{sc}(X)$ has the same homotopy type as $B \operatorname{Aut}^{h}(X)$.

Proof of Theorem 6.2. Consider the category $\mathcal{M}^{[n]}$ of functors $[n] \rightarrow \mathcal{M}$. If $\mathcal{M}$ is a model category, then $\mathcal{M}^{[n]}$ can be given the structure of a model category with the weak equivalences and fibrations given by levelwise weak equivalences and fibrations in $\mathcal{M}$. Given any map $[m] \rightarrow[n]$, we obtain a functor $\mathcal{M}^{[n]} \rightarrow \mathcal{M}^{[m]}$.

Let $Y=\left(y_{0} \rightarrow y_{1} \cdots \rightarrow y_{n}\right)$ be a fibrant-cofibrant object of $\mathcal{M}^{[n]}$. It restricts to an object $Y^{\prime}=\left(y_{0} \rightarrow y_{1} \cdots \rightarrow y_{n-1}\right)$ in $\mathcal{M}^{[n-1]}$. From this map, we obtain a map of simplicial sets

$$
\operatorname{BAut}_{L^{H} \mathcal{M}[n]}^{h}(Y) \rightarrow B \operatorname{Aut}_{L^{H} \mathcal{M}}^{h}\left(y_{n}\right) \times B \operatorname{Aut}_{L^{H} \mathcal{M}^{[n-1]}}^{h}\left(Y^{\prime}\right) .
$$

The homotopy fiber of this map is weakly equivalent to the union of those components of $\operatorname{Map}_{L^{H} \mathcal{M}}\left(y_{n-1}, y_{n}\right)$ containing the conjugates of the map $f_{n-1}: y_{n-1} \rightarrow y_{n}$, or maps $j \circ f_{n-1} \circ i$, where $i$ and $j$ are self-homotopy equivalences.

Iterating this process, we can take the homotopy fiber of the map

$$
\operatorname{BAut}_{L^{H} \mathcal{M}[n]}^{h}(Y) \rightarrow B \operatorname{Aut}_{L^{H} \mathcal{M}}^{h}\left(y_{n}\right) \times \cdots \times B \operatorname{Aut}_{L^{H} \mathcal{M}}^{h}\left(y_{0}\right),
$$

which is weakly equivalent to the union of the components of

$$
\operatorname{Map}_{L^{H} \mathcal{M}}\left(y_{n-1}, y_{n}\right) \times \cdots \times \operatorname{Map}_{L^{H} \mathcal{M}}\left(y_{0}, y_{1}\right)
$$

containing conjugates of the sequence of maps $f_{i}: y_{i} \rightarrow y_{i+1}, 0 \leq i \leq n-1$. However, applying Proposition 6.3 to the map in question shows that this simplicial set is also the homotopy fiber of the map

$$
\operatorname{sc}(Y) \rightarrow \operatorname{sc}\left(y_{n}\right) \times \cdots \times \operatorname{sc}\left(y_{0}\right) .
$$

Let $U$ denote the simplicial space $N(\mathcal{M}, \mathcal{W})$ so that $U_{n}=\operatorname{nerve}\left(\operatorname{we}\left(\mathcal{M}^{[n]}\right)\right)$. Then, let $V$ be a Reedy fibrant replacement of $U$, from which we get weak equivalences $U_{n} \rightarrow V_{n}$ for all $n \geq 0$.

For each $n \geq 0$, there exists a map $p_{n}: U_{n} \rightarrow U_{0}^{n+1}$ given by iterated face maps to the "objects." Then, for every $(n+1)$-tuple of objects $\left(x_{0}, x_{1}, \ldots, x_{n}\right)$, the homotopy fiber of $p_{n}$ over $\left(x_{0}, \ldots, x_{n}\right)$, given by

$$
\operatorname{map}_{V}\left(x_{n-1}, x_{n}\right) \times \cdots \times \operatorname{map}_{V}\left(x_{0}, x_{1}\right),
$$

is weakly equivalent to

$$
\operatorname{Map}_{L^{H} \mathcal{M}}\left(x_{n-1}^{c f}, x_{n}^{c f}\right) \times \cdots \times \operatorname{Map}_{L^{H} \mathcal{M}}\left(x_{0}^{c f}, x_{1}^{c f}\right),
$$

where $x^{c f}$ denotes a fibrant-cofibrant replacement of $X$ in $\mathcal{M}$. It follows that once we take the Reedy fibrant replacement $V$ of $U$, it is a Segal space. 
Now, consider the set $\pi_{0} U_{0}$, which consists of the weak equivalence classes of objects in $\mathcal{M}$; it follows that $\pi_{0} V_{0}$ is an isomorphic set. Further, note that

$$
\operatorname{Hom}_{\text {Ho }(\mathcal{M})}(x, y)=\pi_{0} \operatorname{Map}_{L^{H} \mathcal{M}}\left(x^{c f}, y^{c f}\right) .
$$

Thus, we have shown that $\operatorname{Ho}(\mathcal{M})$ is equivalent to $\operatorname{Ho}(V)$.

It remains to show that $V$ is a complete Segal space. Consider the space $V_{\text {hoequiv }} \subseteq V_{1}$, and define $U_{\text {hoequiv }}$ to be the preimage of $V_{\text {hoequiv }}$ under the natural map $U \rightarrow V$. Since $V$ is a Reedy fibrant replacement for $U$, it suffices to show that the complete Segal space condition holds, i.e., that $U_{0} \rightarrow U_{\text {hoequiv }}$ is a weak equivalence of simplicial sets. Notice that $U_{\text {hoequiv }}$ must consist precisely of the components of $U_{1}$ whose 0 -simplices come from weak equivalences in $\mathcal{M}$. In other words, $U_{\text {hoequiv }}=\operatorname{nerve}\left(\operatorname{we}(\text { we }(\mathcal{M}))^{[1]}\right)$.

There is an adjoint pair of functors

$$
F: \mathcal{M}^{[1]} \rightleftarrows \mathcal{M}: G
$$

for which $F(x \rightarrow y)=x$ and $G(x)=\mathrm{id}_{x}$. This adjoint pair can be restricted to an adjoint pair

$$
F: \operatorname{nerve}\left(\operatorname{we}(\operatorname{we}(\mathcal{M}))^{[1]}\right) \rightleftarrows \operatorname{we}(\mathcal{M}): G,
$$

which in turn induces a weak equivalence of simplicial sets on the nerves, $U_{\text {hoequiv }} \simeq$ $U_{0}$, which completes the proof.

Now that we have proved that the mapping spaces and homotopy categories agree for $V$ and for $L^{H} \mathcal{M}$, it remains to show that they agree for $L^{H} \mathcal{M}$ and $L_{C}\left(L^{H} \mathcal{M}\right)$.

Theorem 6.4. Let $\mathcal{M}$ be a model category. For any $x$ and $y$ objects of $L^{H} \mathcal{M}$, there is a weak equivalence of simplicial sets

$$
\operatorname{Map}_{L^{H} \mathcal{M}}(x, y) \simeq \operatorname{map}_{L_{C}\left(L^{H} \mathcal{M}\right)}(x, y),
$$

and there is an equivalence of categories $\pi_{0} L^{H} \mathcal{M} \approx \operatorname{Ho}\left(L_{C}\left(L^{H} \mathcal{M}\right)\right)$.

Note in particular that $x$ and $y$ are just objects of $\mathcal{M}$, and that $\pi_{0} L^{H} \mathcal{M}$ is equivalent to the homotopy category $\operatorname{Ho}(\mathcal{M})$.

Proof. Given the hammock localization $L^{H} \mathcal{M}$ of the model category $\mathcal{M}$, we have the following composite map of simplicial spaces:

$$
X=\operatorname{nerve}\left(L^{H} \mathcal{M}\right) \rightarrow X^{f} \rightarrow L_{C}\left(L^{H} \mathcal{M}\right) .
$$

Here, $X^{f}$ denotes a Reedy fibrant replacement of $X$. This composite is just Rezk's method for assigning the complete Segal space $L_{C}\left(L^{H} \mathcal{M}\right)$ to the simplicial category $L^{H} \mathcal{M}$.

On the left-hand side, the mapping spaces of $X=\operatorname{nerve}\left(L^{H} \mathcal{M}\right)$ are precisely those of $L^{H} \mathcal{M}$, by the definition of the nerve functor. In the nerve, one of these mapping spaces, say $\operatorname{map}_{X}(x, y)$ for some objects $x$ and $y$ of $\mathcal{M}$, is given by the fiber over $(x, y)$ of the map $\left(d_{1}, d_{0}\right): X_{1} \rightarrow X_{0} \times X_{0}$. Although these mapping spaces can be defined for $X$, there is no reason that they are homotopy invariant. When we take a Reedy fibrant replacement $X^{f}$ of $X$, however, this map becomes a fibration, and hence this fiber is actually a homotopy fiber and so homotopy invariant. For a general simplicial space, we cannot assume that the mapping spaces of the Reedy fibrant replacement are equivalent to the original ones. However, if the 0-space of the simplicial space in question is discrete in degree zero, then the map above is a fibration. Using an argument similar to the one in [5, §5], we can find a Reedy 
fibrant replacement functor which leaves the 0-space discrete. While the space in degree one might be changed in this process of passing to $X_{1}^{f}$, it will still be weakly equivalent to $X_{1}$. In particular, the mapping spaces in $X^{f}$ will be weakly equivalent to those in $X$.

Since the objects of $X^{f}$ are just the objects of $L^{H} \mathcal{M}$, or the objects of $\mathcal{M}$, this equivalence of mapping spaces gives us also an equivalence of homotopy categories.

The right-most map is the one defined by Rezk, $i_{X^{f}}: X^{f} \rightarrow \widehat{X^{f}}$, which takes a Segal space to a complete Segal space. But, he defines this map in such a way that it is in fact a Dwyer-Kan equivalence. In other words, it induces weak equivalences on mapping spaces and an equivalence of homotopy categories. Thus, the composite map induces equivalences on mapping spaces and an equivalence on homotopy categories.

\section{A characterization of Complete Segal spaces ARISING FROM SIMPLICIAL CATEGORIES}

In this section, we give a thorough description of the weak equivalence type of complete Segal spaces which occur as images of Rezk's functor from the category of simplicial categories. We consider several different cases, beginning with ones for which we can use the classifying diagram construction, i.e., discrete categories, and then proceed to more general simplicial categories.

It should be noted that we are characterizing these complete Segal spaces up to weak equivalence, and so the resulting descriptions are of the homotopy type of the spaces in each simplicial degree. For example, in the case of a discrete category, we describe the corresponding complete Segal space in terms of the isomorphism classes of objects, rather than in terms of individual objects, in order to simplify the description. One could just take all objects, and generally get much larger spaces, if the more precise description were needed for the complete Segal space corresponding to a given category.

Furthermore, notice that determining the homotopy type of the spaces in degrees zero and one is sufficient to determine the homotopy type of all the spaces, since we are considering Segal spaces. Thus, we focus our attention on these spaces, adding in a few comments about how to continue the process with the higher-degree spaces.

7.1. Case 1: $\mathcal{C}$ is a discrete groupoid. If $\mathcal{C}=G$ is a group, then applying Rezk's classifying diagram construction results in a complete Segal space equivalent to $B G$, i.e., the constant simplicial space which is the simplicial set $B G$ at each level. In particular, since all morphisms are invertible, we obtain essentially no new information at level 1 that we didn't have already at level 0 .

Example 7.1. Let $G=\mathbb{Z} / 2$. Then $(N G)_{0}$ is just the nerve, or $B \mathbb{Z} / 2$. Then $(N G)_{1}$ has two 0 -simplices, given by the two morphisms (elements) of $G$. However, these two objects of $G^{[1]}$ are isomorphic, and the automorphism group of either one of them is $\mathbb{Z} / 2$. Thus, $(N G)_{1}$ is also equivalent to $B \mathbb{Z} / 2$.

If $\mathcal{C}$ has more than one object but only one isomorphism class of objects, we get instead a simplicial space weakly equivalent to the constant simplicial space which is $B \operatorname{Aut}(x)$ at each level, for a representative object $x$. If $\mathcal{C}$ has more than one isomorphism class $\langle x\rangle$, then the result will be weakly equivalent to the constant simplicial space $\coprod_{\langle x\rangle} B \operatorname{Aut}(x)$. 
7.2. Case 2: $\mathcal{C}$ is a discrete category. Since in the classifying diagram $N \mathcal{C}$, $(N \mathcal{C})_{0}$ picks out the isomorphisms of $\mathcal{C}$ only, we still essentially have $\coprod_{\langle x\rangle} B \operatorname{Aut}(x)$ at level 0 . However, if $\mathcal{C}$ is not a groupoid, then there is new information at level 1. It instead looks like

$$
\coprod_{\langle x\rangle,\langle y\rangle} B \operatorname{Aut}\left(\coprod_{\langle\alpha\rangle} \operatorname{Hom}(x, y)_{\alpha}\right)
$$

where the $\alpha$ indexes the isomorphism classes of elements of $\operatorname{Hom}(x, y)$. The subspace of $(N \mathcal{C})_{1}$ corresponding to $\langle x\rangle,\langle y\rangle$, denoted $(N \mathcal{C})_{1}(x, y)$, fits into a fibration

$$
\operatorname{Hom}(x, y) \rightarrow(N \mathcal{C})_{1}(x, y) \rightarrow B \operatorname{Aut}(x) \times B \operatorname{Aut}(y) .
$$

The space in dimension 2 is determined, then, by the spaces at levels 0 and 1 . The subspace corresponding to isomorphism classes of objects $\langle x\rangle,\langle y\rangle,\langle z\rangle$, denoted $(N \mathcal{C})_{2}(x, y, z)$, fits into a fibration

$$
\operatorname{Hom}(x, y) \times \operatorname{Hom}(y, z) \rightarrow(N \mathcal{C})_{2}(x, y, z) \rightarrow B \operatorname{Aut}(x) \times B \operatorname{Aut}(y) \times B \operatorname{Aut}(z) .
$$

The whole space $(N \mathcal{C})_{2}$, up to homotopy, looks like

$$
\coprod_{\langle x\rangle,\langle y\rangle,\langle z\rangle} B \operatorname{Aut}\left(\coprod_{\langle\alpha\rangle,\langle\beta\rangle} \operatorname{Hom}(x, y)_{\alpha} \times \operatorname{Hom}(y, z)_{\beta}\right) .
$$

We could describe each $(N \mathcal{C})_{n}$ analogously.

Example 7.2. Let $\mathcal{C}$ denote the category with two objects and one nontrivial morphism between them $(\cdot \rightarrow \cdot)$. If $\{e\}$ denotes the trivial group, then $(N \mathcal{C})_{0} \simeq$ $B\{e\} \amalg B\{e\}$ and $(N \mathcal{C})_{1} \simeq B\{e\} \amalg B\{e\} \amalg B\{e\}$. In particular, $N \mathcal{C}$ is not equivalent to the classifying diagram of the trivial category with one object and one morphism, which would be the constant simplicial space $B\{e\}$. However, note that the nerves of these two categories are homotopy equivalent. Thus, we can see that the classifying diagram is more refined than the nerve in distinguishing between these two categories.

7.3. Case 3: $\mathcal{C}$ is a simplicial groupoid. First, consider the case where we have a simplicial group $G$. Let $G_{n}$ denote the group of $n$-simplices of $G$. Then

$$
\operatorname{hocolim}_{\Delta^{\circ p}}\left(\operatorname{nerve}\left(G_{n}\right)^{t}\right)=\operatorname{nerve}(G) \text {. }
$$

Let $L_{C}$ denote Rezk's completion functor, which makes the nerve into a complete Segal space. We claim that

$$
L_{C}\left(\operatorname{hocolim}_{\Delta^{o p}}\left(\operatorname{nerve}\left(G_{n}\right)^{t}\right)\right) \simeq L_{C}\left(\operatorname{hocolim}_{\Delta^{\circ p}} L_{C}\left(\operatorname{nerve}\left(G_{n}\right)^{t}\right)\right) .
$$

We actually prove the more general statement that, for any $X=\operatorname{hocolim}_{\Delta^{o p}} X_{n}$,

$$
L_{C}\left(\operatorname{hocolim}_{\Delta}{ }^{o p} X_{n}\right) \simeq L_{C}\left(\text { hocolim }_{\Delta}{ }^{o p} L_{C} X_{n}\right) .
$$

To prove this claim, first note that we have Rezk's completion map

$$
i: \operatorname{hocolim}_{\Delta}{ }^{o p} X_{n} \rightarrow L_{C}\left(\text { hocolim }_{\Delta}{ }^{o p} X_{n}\right),
$$

which is a weak equivalence. Furthermore, since in $\mathcal{C S S}$ any complete Segal space $Y$ is a local object and every object is cofibrant, we have a weak equivalence of spaces

$$
\operatorname{Map}\left(L_{C}\left(\operatorname{hocolim}_{\Delta^{o p}} X_{n}\right), Y\right) \simeq \operatorname{Map}\left(\operatorname{hocolim}_{\Delta^{o p}} X_{n}, Y\right) .
$$


So, for any complete Segal space $Y$, we have that

$$
\begin{aligned}
\operatorname{Map}\left(L_{C} \operatorname{hocolim}_{\Delta^{o p}}\left(L_{C} X_{n}\right), Y\right) & \simeq \operatorname{Map}\left(\operatorname{hocolim}_{\Delta}{ }^{o p}\left(L_{C} X_{n}\right), Y\right) \\
& \left.\simeq \operatorname{holim} \operatorname{Map}_{C} L_{n}, Y\right) \\
& \simeq \operatorname{holim} L_{\Delta} \operatorname{Map}\left(X_{n}, Y\right) \\
& \simeq \operatorname{Map}\left(\operatorname{hocolim}_{\Delta}{ }^{o p} X_{n}, Y\right) \\
& \simeq \operatorname{Map}\left(L_{C} \operatorname{hocolim}_{\Delta}{ }^{o p} X_{n}, Y\right) .
\end{aligned}
$$

Note that the above calculation depends on the fact that

$$
\operatorname{Map}\left(\operatorname{hocolim}_{\Delta}{ } X_{n}, Y\right) \simeq \operatorname{holim}_{\Delta} \operatorname{Map}\left(X_{n}, Y\right)
$$

which follows from working levelwise on simplicial sets.

Then, since $G_{n}$ is a discrete group, completing its nerve is the same as taking the classifying diagram $N G_{n}$ which, by case 1 , is weakly equivalent to the constant simplicial space $B G_{n}$, denoted here $c B G_{n}$. Thus we have:

$$
\begin{aligned}
L_{C}(\operatorname{nerve}(G)) & \simeq L_{C}\left[\operatorname{hocolim} \Delta^{o p}\left(\operatorname{nerve}\left(G_{n}\right)\right)\right] \\
& \simeq L_{C}\left[\operatorname{hocolim} \Delta^{o p}\left(L_{C}\left(\operatorname{nerve}\left(G_{n}\right)\right)\right)\right] \\
& \simeq L_{C}\left[\operatorname{hocolim} \boldsymbol{\Delta}^{o p}\left(c B G_{n}\right)\right] \\
& \simeq L_{C}(B G) \\
& \simeq B G .
\end{aligned}
$$

So, we obtain a simplicial space weakly equivalent to the constant simplicial space with $B G$ at each level. (Recall, however, that $B G$ here is obtained by taking the diagonal of the simplicial nerve, so it is not quite the identical case.) If we have a simplicial groupoid, rather than a simplicial group, we obtain the analogous result, replacing $B G$ with

$$
\coprod_{\langle x\rangle} B \operatorname{Aut}(x) .
$$

7.4. Case 4: $\mathcal{C}$ is a simplicial category with every morphism invertible up to homotopy. Alternatively stated, this case covers the situation in which $\pi_{0}(\mathcal{C})$ is a groupoid.

Recall that we have a model structure $\mathcal{S C}_{\mathcal{O}}$ on the category of categories with a fixed object set $\mathcal{O}$, in which the cofibrant objects are retracts of free objects. So, taking a cofibrant replacement of $\mathcal{C}$ in this model category structure $\mathcal{S C}_{\mathcal{O}}$ essentially gives a free replacement of $\mathcal{C}$, denoted $F(\mathcal{C})$, which is weakly equivalent to $\mathcal{C}$. (This cofibrant category can be obtained by taking a simplicial resolution $F_{*} \mathcal{C}$ and then taking a diagonal [12, 6.1].) Now, taking the localization with respect to all morphisms results in a simplicial groupoid. So, we have Dwyer-Kan equivalences

$$
F(\mathcal{C})^{-1} F(\mathcal{C}) \stackrel{\simeq}{\longleftarrow} F(\mathcal{C}) \stackrel{\simeq}{\longrightarrow} \mathcal{C} .
$$

But, now $F(\mathcal{C})^{-1} F(\mathcal{C})$ is a simplicial groupoid weakly equivalent to $\mathcal{C}$, so we have now reduced this situation to case 3 .

Note that, to write down a description of this complete Segal space in terms of the original category $\mathcal{C}$, we need to take isomorphism classes of objects in $\pi_{0}(\mathcal{C})$, or weak equivalence classes, as well as self-maps which are invertible up to homotopy rather than strict automorphisms. While we will still use $\langle x\rangle$ to denote the equivalence class of a given object, we will use $\operatorname{Aut}^{h}(x)$ to signify homotopy automorphisms of 
$x$. Thus, the complete Segal space corresponding to $\mathcal{C}$ in this case essentially looks like

$$
\coprod_{\langle x\rangle} B \operatorname{Aut}^{h}(x)
$$

at each level.

7.5. Case 5: $\mathcal{C}$ is any simplicial category. First consider the subcategory of $\mathcal{C}$ containing all the objects of $\mathcal{C}$ and only the morphisms of $\mathcal{C}$ which are invertible up to homotopy. Apply case 5 to get a complete Segal space, but take only the 0 -space of it to be the 0 -space of the desired complete Segal space.

To find the 1-space, first recall the definition of the completion functor as applied to a Segal space $W$ :

$$
L_{C}(W)=L_{\mathcal{C S S}}\left(\operatorname{diag}\left([m] \mapsto\left(W^{E(m)}\right)_{n}\right)\right) .
$$

Recall further that $\left(W^{E(m)}\right)_{n}=\operatorname{Map}\left(E(m) \times \Delta[n]^{t}, W\right)$. Thus, the Segal space we obtain (before applying the functor $L_{\mathcal{C S S}}$ ) looks like

$$
\operatorname{Map}\left(E(0) \times \Delta[0]^{t}, W\right) \Leftarrow \operatorname{Map}\left(E(1) \times \Delta[1]^{t}, W\right) \Leftarrow \operatorname{Map}\left(E(2) \times \Delta[2]^{t}, W\right) \cdots .
$$

If the Segal space $W$ is a fibrant replacement of nerve $(\mathcal{C})$, then the space at level 1 consists of diagrams

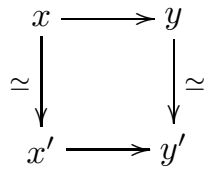

with the maps in the appropriate simplicial level.

For simplicity, we restrict to a given pair of objects $x$ and $y$, representing given equivalence classes. Consider the homotopy automorphisms of $x$ and $y$. If they are not all invertible, we take a cofibrant replacement and group completion as in case 4. So, without loss of generality, assume that $\operatorname{Aut}(x)$ and $\operatorname{Aut}(y)$ are simplicial groups. Note that we have

$$
\operatorname{Aut}(x)=\operatorname{hocolim}_{\Delta^{o p}} \operatorname{Aut}(x)_{n}
$$

and

$$
\operatorname{Aut}(y)=\operatorname{hocolim}_{\Delta^{o p}} \operatorname{Aut}(y)_{n}
$$

Now look at

$$
\operatorname{Map}(x, y)=\operatorname{hocolim}_{\Delta^{o p}} \operatorname{Map}(x, y)_{n} .
$$

Consider for each $n \geq 0$ the discrete category $\mathcal{C}(x, y)_{n}$ which has as objects $\operatorname{Map}(x, y)_{n}$ and as morphisms pairs $(\alpha, \beta)$ of automorphisms in $\operatorname{Aut}(x)_{n} \times \operatorname{Aut}(y)_{n}$ making a commutative square

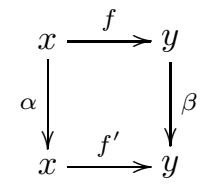

with $f, f^{\prime} \in \operatorname{Map}(x, y)_{n}$.

Thus, the 1-space that we are interested in is also the 1-space of the complete Segal space given by

$$
L_{\mathcal{C S S}}\left(\operatorname{hocolim}_{\boldsymbol{\Delta}^{o p}}\left(\operatorname{nerve}\left(\mathcal{C}(x, y)_{n}\right)\right)\right) .
$$


Using a straightforward argument about localization functors similar to the one in case 3 (which can be found in [3, 4.1]), we can also apply the functor $L_{\mathcal{C S S}}$ on the inside to get an equivalent simplicial space

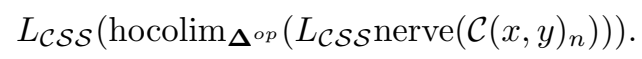

But, since $\mathcal{C}(x, y)_{n}$ is a discrete category, this space is just

$$
L_{\mathcal{C S S}} \operatorname{hocolim}_{\boldsymbol{\Delta}^{o p}}\left(N \mathcal{C}(x, y)_{n}\right) \simeq \operatorname{hocolim}_{\boldsymbol{\Delta}^{o p}}\left(N \mathcal{C}(x, y)_{n}\right) .
$$

Now, we restrict to the 1 -space here, which is

$\operatorname{hocolim}_{\Delta^{o p}}\left(\coprod_{\langle x\rangle,\langle y\rangle} B \operatorname{Aut}\left(\coprod_{\langle\alpha\rangle} \operatorname{Map}_{n}(x, y)_{\alpha}\right)\right) \simeq \coprod_{\langle x\rangle,\langle y\rangle} B \operatorname{Aut}\left(\coprod_{\langle\alpha\rangle} \operatorname{Map}(x, y)_{\alpha}\right)$.

As with the previous case, we can then go back and weaken to homotopy automorphisms and equivalence classes of objects to consider categories before taking a group completion, so our space looks like

$$
\coprod_{\langle x\rangle,\langle y\rangle} B^{\operatorname{Aut}^{h}}\left(\coprod_{\langle\alpha\rangle} \operatorname{Map}(x, y)_{\alpha}\right)
$$

We could then obtain the 2-space of our complete Segal space by considering categories $\mathcal{C}(x, y, z)_{n}$ defined similarly, and the description of the 2 -space of the classifying diagram of a discrete category as given in case 2 .

We can summarize these results in the following theorem. For an object $x$ of a simplicial category $\mathcal{C}$, let $\langle x\rangle$ denote the weak equivalence class of $x$ in $\mathcal{C}$, and for a morphism $\alpha: x \rightarrow y$, let $\langle\alpha\rangle$ denote the weak equivalence class of $\alpha$ in the morphism category $\mathcal{C}^{[1]}$. Let $\operatorname{Aut}^{h}(x)$ denote the space of self-maps of $x$ which are invertible in $\pi_{0} \mathcal{C}$.

Theorem 7.3. Let $\mathcal{C}$ be a simplicial category. The complete Segal space corresponding to $\mathcal{C}$ has the form

$$
\coprod_{\langle x\rangle} B \operatorname{Aut}^{h}(x) \Leftarrow \coprod_{\langle x\rangle,\langle y\rangle} B \operatorname{Aut}^{h}\left(\coprod_{\langle\alpha\rangle} \operatorname{Map}(x, y)_{\alpha}\right) \Leftarrow \cdots .
$$

\section{ACKNOWLEDGMENTS}

The author would like to thank Bill Dwyer, Charles Rezk, André Joyal, and Myles Tierney for conversations about the work in this paper, as well as the referee for helpful comments on the exposition.

\section{REFERENCES}

[1] J.E. Bergner, Homotopy fiber products of homotopy theories, in preparation.

[2] J.E. Bergner, A model category structure on the category of simplicial categories, Trans. Amer. Math. Soc. 359 (2007), 2043-2058. MR2276611(2007i:18014)

[3] J.E. Bergner, Simplicial monoids and Segal categories, Contemp. Math. 431 (2007), 59-83. MR.2342822

[4] J.E. Bergner, A survey of $(\infty, 1)$-categories, preprint available at math.AT/0610239.

[5] J.E. Bergner, Three models for the homotopy theory of homotopy theories, Topology 46 (2007), 397-436. MR2321038

[6] J.M. Cordier and T. Porter, Vogt's theorem on categories of homotopy coherent diagrams, Math. Proc. Camb. Phil. Soc. (1986), 100, 65-90. MR838654(87i:55027) 
[7] Daniel Dugger, Universal homotopy theories, Adv. Math. 164 (2001), no. 1, 144-176. MR1870515 (2002k:18021)

[8] W.G. Dwyer and D.M. Kan, Calculating simplicial localizations, J. Pure Appl. Algebra 18 (1980), 17-35. MR:578563(81h:55019)

[9] W.G. Dwyer and D.M. Kan, A classification theorem for diagrams of simplicial sets, Topology 23(1984), 139-155. MR744846 (86c:55010a)

[10] W.G. Dwyer and D.M. Kan, Equivalences between homotopy theories of diagrams, Algebraic topology and algebraic K-theory (Princeton, N.J., 1983), 180-205, Ann. of Math. Stud., 113, Princeton Univ. Press, Princeton, NJ, 1987. MR921478 (89d:55051)

[11] W.G. Dwyer and D.M. Kan, Function complexes in homotopical algebra, Topology 19 (1980), 427-440. MR584566 (81m:55018)

[12] W.G. Dwyer and D.M. Kan, Simplicial localizations of categories, J. Pure Appl. Algebra 17 (1980), no. 3, 267-284. MR579087 (81h:55018)

[13] W.G. Dwyer and J. Spalinski, Homotopy theories and model categories, in Handbook of Algebraic Topology, North-Holland, Amsterdam, 1995. MR1361887(96h:55014)

[14] P.G. Goerss and J.F. Jardine, Simplicial Homotopy Theory, Progress in Math, vol. 174, Birkhäuser, 1999. MR.1711612 (2001d:55012)

[15] Philip S. Hirschhorn, Model Categories and Their Localizations, Mathematical Surveys and Monographs 99, AMS, 2003. MR1944041 (2003j:18018)

[16] Mark Hovey, Model Categories, Mathematical Surveys and Monographs, 63. American Mathematical Society 1999. MR1650134 (99h:55031)

[17] A. Joyal, Simplicial categories vs quasi-categories, in preparation.

[18] A. Joyal, The theory of quasi-categories I, in preparation.

[19] André Joyal and Myles Tierney, Quasi-categories vs Segal spaces, Contemp. Math. 431 (2007), 277-326. MR2342834

[20] Saunders Mac Lane, Categories for the Working Mathematician, Second Edition, Graduate Texts in Mathematics 5, Springer-Verlag, 1997. MR1712872 (2001j:18001)

[21] J.P. May, Simplicial Objects in Algebraic Topology, University of Chicago Press, 1967. MR0222892 (36:5942)

[22] C.L. Reedy, Homotopy theory of model categories, unpublished manuscript, available at http://www-math.mit.edu/ psh.

[23] Charles Rezk, A model for the homotopy theory of homotopy theory, Trans. Amer. Math. Soc. 353(3) (2001), 973-1007. MR1804411(2002a:55020)

[24] Bertrand Toën, Derived Hall algebras, Duke Math. J. 135, no. 3 (2006), 587-615. MR 2272977 (2007h:18021)

Department of Mathematics, Kansas State University, 138 Cardwell Hall, ManhatTAN, KANSAS 66506

E-mail address: bergnerj@member.ams.org

Current address: Department of Mathematics, University of California, Riverside, Riverside, California 92521 